
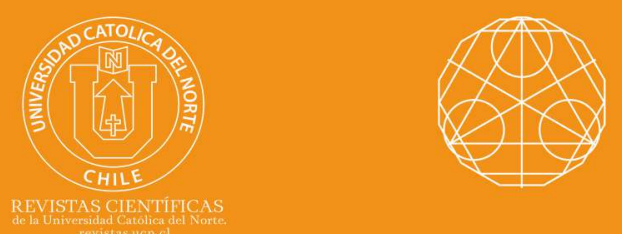

\title{
Strong Riesz summability of Fourier series
}

Bidu Bhusan Jena' ${ }^{1}$ orcid.org/0000-0001-6776-0993

Susanta Kumar Paikray ${ }^{2}$ (10 orcid.org/0000-0003-4465-7621

Umakanta Misra ${ }^{3}$ (1) orcid.org/0000-0002-9989-1047

Veer Surendra Sai University of Technology, Dept. of Mathematics, Burla, OR, India. $1 \llbracket$ bidumath.05@gmail.com; 2 skpaikray_math@vssut.ac.in

${ }^{3}$ National Institute of Science and Technology, Dept. of Mathematics, Golanthara, OR, India umakanta_misra@yahoo.com

Received: January 2019 | Accepted: August 2020

\section{Abstract:}

The notion of strong summability was introduced by Fekete (Math. És Termesz Ertesitö, 34 (1916), 759-786). Dealing with Nörlund summability of Fourier series Mittal (J. Math. Anal. Appl. 314 (2006), 75-84) has established a result on strong summability. We have established a new result on sufficient condition for strong Riesz summability of Fourier series.

Keywords: Strong summability; Cesàro summability; $\left[\bar{N}, p_{n}^{(1)}, 2\right]$ summability; Fourier series.

MSC (2020): Primary 40F05, Secondary 40G05.

\section{Cite this article as (IEEE citation style):}

B. B. Jena, S. K. Paikray, and U. Misra, "Strong Riesz summability of Fourier series", Proyecciones (Antofagasta, On line), vol. 39, no. 6, pp. 1615-1626, Dec. 2020, doi: 10.22199/issn.0717-62792020-06-0096.

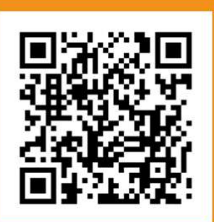

Article copyright: (C) 2020 Bidu Bhusan Jena, Susanta Kumar Paikray, and Umakanta Misra. This is an open access article distributed under the terms of the Creative Commons License, which permits unrestricted use and distribution provided the original author and source are credited. 


\section{Introduction}

Let $\sum u_{n}$ be an infinite series with a sequence of partial sum $\left(s_{n}\right)$. The sequence-to-sequence transformation,

$$
C_{n}^{\alpha}=\frac{1}{E_{n}^{\alpha-1}} \sum_{r=0}^{n} s_{n-r} E_{r}^{\alpha-2}
$$

where

$$
E_{n}^{\alpha-1}=\frac{\Gamma(n+1)}{\Gamma(n+1) \Gamma(\alpha)}, \alpha>0,
$$

defines the Cesàro mean of order $\alpha$ of $\left(s_{n}\right)$. The series $\sum u_{n}$ is said to be summable $(C, \alpha)$ to the sum $s$ if,

$$
\lim _{n \rightarrow \infty} C_{n}^{\alpha}=s
$$

and is said to be strongly summable $(C, \alpha)$ with index $q$ or $[C, \alpha, q], \alpha>$ $0, q>0$, to the sum $s$ (see [4]), if

$$
\sum_{r=0}^{n}\left|C_{r}^{\alpha-1}-s\right|^{q}=o(n)(n \rightarrow \infty) .
$$

Let $\left(p_{n}\right)$ be a sequence of non-negative numbers such that

$$
P_{n}=\sum_{r=0}^{n} p_{r},\left(P_{-1}=p_{-1}=0\right), p_{0}=0 .
$$

The sequence to sequence transformation

$$
t_{n}=\frac{1}{P_{n}} \sum_{r=0}^{n} s_{r} p_{n-r}, n=0,1,2, \ldots,
$$

defines the sequence $\left(t_{n}\right)$ of the $\left(N, p_{n}\right)$ mean of the sequence $\left(s_{n}\right)$ generated by the sequence of coefficients $\left(p_{n}\right)$. The series $\sum u_{n}$ is said to be strongly summable $\left(N, p_{n}\right)$ with index $q$ or $\left[N, p_{n}, q\right], q>0$, to the sum $s$ (see [14]), if

$$
\frac{1}{\left|P_{n}\right|} \sum_{r=0}^{n}\left|p_{r}\right|\left|t_{r}^{\prime}-s\right|^{q}=o(1)
$$

where 


$$
\begin{aligned}
t_{r}^{\prime} & =\frac{1}{P_{n}} \sum_{r=0}^{n} s_{r} \nabla p_{n-r},\left(\nabla p_{n-r} \equiv p_{n-r}-p_{n-r-1}\right) \\
& =\frac{1}{p_{n}} \sum_{r=0}^{n} u_{r} p_{n-r} .
\end{aligned}
$$

Here, just by taking

$$
p_{n}^{\alpha}=\sum_{k=0}^{n} E_{n-k}^{\alpha-1} p_{k}
$$

in place of $p_{n}$; it can further be extended to $\left[N, p_{n}^{\alpha}, q\right], \alpha \geq 1, q \geq 1$, summability. It is noticed that putting $p_{n}=E_{n}^{\alpha-1}$ in (1.1), $\left(t_{n}\right)$ reduces to the Cesàro mean of order $\alpha$ of $\left(s_{n}\right)$.

Similarly, the sequence to sequence transformation,

$$
T_{n}=\frac{1}{P_{n}} \sum_{r=0}^{n} s_{r} p_{r}
$$

defines $\left(\bar{N}, p_{n}\right)$ mean of the sequence $\left(s_{n}\right)$ generated by the sequence of coefficients $\left(p_{n}\right)$. The series $\sum u_{n}$ is said to be strongly summable $\left(\bar{N}, p_{n}\right)$ with index $q$ or $\left[\bar{N}, p_{n}, q\right], q>0$, to the sum $s$, if

$$
\frac{1}{\left|P_{n}\right|} \sum_{r=0}^{n}\left|p_{r}\right|\left|T_{r}^{\prime}-s\right|^{q}=o(1)
$$

where

$$
\begin{aligned}
T_{r}^{\prime} & =\frac{1}{P_{n}} \sum_{r=0}^{n} s_{r} \nabla p_{r},\left(\nabla p_{r} \equiv p_{r}-p_{r-1}\right) \\
& =\frac{1}{p_{n}} \sum_{r=0}^{n} u_{r} p_{r} .
\end{aligned}
$$

Similar to $\left[N, p_{n}^{\alpha}, q\right](\alpha \geq 1, q \geq 1)$-summability, $\left[\bar{N}, p_{n}^{\alpha}, q\right](\alpha \geq 1, q \geq 1)$ summability can also be defined.

Let $f(t)$ be a $2 \pi$ - periodic function such that $f \in L[-\pi, \pi]$. Let $s[f]$ denote the Fourier series of $f(t)$, given by

$$
s[f]=\left(a_{0} / 2\right)+\sum_{n=1}^{\infty}\left(a_{n} \cos n t+b_{n} \sin n t\right)=\sum_{n=1}^{\infty} u_{n}(t)
$$

with a sequence of its partial sums $\left(s_{n}(t)\right)$, where the constant term $\left(a_{0} / 2\right)$ is assumed to be zero. Moreover, for local property of factored Fourier 
series one may refer to the recent work [7].

We use the following notations:

$$
\begin{aligned}
& \phi(t) \quad \phi_{x}(t)=(1 / 2)[f(x+t)+f(x-t)-2 s] ; \quad v_{r}=r p_{r} / p_{r}^{(1)}=r p_{r} / P_{r} ; \\
& \Phi(t)=\int_{0}^{t}|\phi(u)| d u ; \quad \bar{N}_{n}(t)=\left(\pi P_{n}\right)^{-1} \sum_{r=0}^{n} p_{r} \sin (n+1 / 2) t / \sin (t / 2) ; \\
& \alpha(t)=\sum_{r=0}^{n} p_{r} \cos (r t) ; \quad \beta(t)=\sum_{r=0}^{n} p_{r} \sin (r t) ; \\
& I_{1}=\sum_{m=0}^{r} \frac{p_{m}}{P_{r}}\left(\frac{1}{\pi} \int_{0}^{1 / r} \phi(t) \frac{\sin (m+1 / 2) t}{\sin t / 2}\right) d t ; \\
& I_{2}=\sum_{m=0}^{r} \frac{p_{m}}{P_{r}}\left(\frac{1}{\pi} \int_{1 / r}^{\delta} \phi(t) \frac{\sin (m+1 / 2) t}{\sin t / 2}\right) d t ; \\
& I_{3}=\sum_{m=0}^{r} \frac{p_{m}}{P_{r}}\left(\frac{1}{\pi} \int_{\delta}^{\pi} \phi(t) \frac{\sin (m+1 / 2) t}{\sin t / 2}\right) d t ; \\
& l_{0}=x+1 ; \quad l_{1}=\log (x+1) ; \quad l_{2}(x)=\log \log (x+1), \ldots \text { and so on }, \\
& \text { for } x>0 ;
\end{aligned}
$$

and $\tau=[1 / t]$, the largest integer less than or equal to $1 / t$.

\section{Preliminaries}

The idea of strong summability was introduced by Fekete [2], based on a result of Hardy and Littlewood [3] concerning the summability of Fourier series. A few researchers have used strong summability method in the course of their research works. Moreover, Matrix summability or matrix transformation is very important in the study of summability theory in the sense that it generalizes the different summability methods like Cesàro summability, Nörlund summability, Riesz summability etc. Also, the statistical convergene and statistical summability are more general than the ordinary convergene and ordinary summability. For recent works in this direction, see [1], [5], [6], [8], [9], [10], [11], [15], [16], [17], [18], [19] and [20]. Dealing with strong summability of Nörlund mean Mittal [13] has proved a theorem as follows.

Theorem 1. (see [13]) If

$$
\Phi(t)=o\left(t / \sqrt{\prod_{m=1}^{k+1} l_{m}(1 / t)}\right) \text {, as } t \rightarrow 0
$$


then the series $\sum_{n=0}^{\infty} u_{n}(t)$ is $\left[N, p_{n}^{(1)}, 2\right]$ - summable to the sum $s$ (same sum), for $t=x$, provided

$$
p_{n}=\left[\prod_{m=0}^{k} l_{m}(n)\right]^{-1}, k=0,1,2, \cdots,
$$

holds true.

\section{Main Theorem}

In an attempt to have further studies in this direction we have established a criterion for the $\left[\bar{N}, p_{n}^{(1)}, 2\right]$ summability and established a new theorem on sufficient condition for strong Riesz summability of Fourier series, as follows.

\section{Theorem 1. If}

$$
\Phi(t)=o\left(t / \sqrt{\prod_{m=1}^{k+1} l_{m}(1 / t)}\right)(t \rightarrow 0),
$$

then the series $\sum_{n=0}^{\infty} u_{n}(t)$ is strongly $\left[\bar{N}, p_{n}^{(1)}, 2\right]$-summable to the sum $s$ (same sum), for $t=x$, provided

$$
p_{n}=\left[\prod_{m=0}^{k} l_{m}(n)\right]^{-1}, k=0,1,2, \cdots,
$$

holds true.

\section{Required Lemmas}

We need to prove the following lemmas for the proof of our theorem.

Lemma 1. If

$$
\Phi(t)=o(t), \quad t \rightarrow 0
$$

then,

$$
I_{1}=o(1) \text { and } I_{3}=o(1)
$$


Proof. By using Riemann - Lebesgue lemma and the regularity condition of summation, $I_{3}=o(1)$. Next, since $\bar{N}_{r}(t)=O(r)$ is uniform in $[0,1 / r]$, so by $(4.1)$ we have

$$
\begin{aligned}
I_{1} & =O\left[\int_{0}^{1 / r}|\phi(t)| \bar{N}_{r}(t) \mid d t\right] \\
& =O(r) \Phi(1 / r) \\
& =O(r) o(1 / r)=o(1) \quad(t \rightarrow 0) .
\end{aligned}
$$

Lemma 2. Let $\left\{p_{n}\right\}$ is a non-negative and non-increasing sequence. If condition (4.1) of Lemma 1 holds true, then

$$
\begin{aligned}
I_{2}=\frac{1}{\pi P_{r}} \int_{1 / r}^{\delta} \phi(t) \alpha(t) d t \\
\quad+\frac{1}{\pi P_{r}} \int_{1 / r}^{\delta} \phi(t) \beta(t) \cot (t / 2) d t+o(1) .
\end{aligned}
$$

Proof. Since $p_{r} \geq p_{r+1}, v_{n}=O(1)$, so we have

$$
\begin{aligned}
I_{2}= & \frac{1}{\pi P_{r}} \int_{1 / r}^{\delta} \phi(t)\left[\sum_{m=0}^{\tau}+\sum_{m=\tau+1}^{r}\right] p_{m} \frac{\sin (m+1 / 2) t}{\sin (t / 2)} d t \\
= & \frac{1}{\pi P_{r}} \int_{1 / r}^{\delta} \frac{\phi(t)}{\sin (t / 2)}[\alpha(t) \sin (t / 2)+\beta(t) \cos (t / 2)] d t \\
& +\frac{1}{\pi P_{r}} \int_{1 / r}^{\delta} \frac{\phi(t)}{\sin (t / 2)}\left[\sum_{m=\tau+1}^{r} p_{m} \sin (m+1 / 2) t\right] d t \\
= & I_{2,1}+I_{2,2} \quad \text { (say). }
\end{aligned}
$$
have,

Thus for proving the validity of (2) it is suffices to show $I_{2,2}=o(1)$. We

$$
\begin{aligned}
I_{2,2} & =\frac{1}{\pi P_{r}} \int_{1 / r}^{\delta} \frac{\phi(t)}{\sin (t / 2)}\left[\sum_{m=\tau+1}^{r} p_{m} \sin (m+1 / 2) t\right] d t \\
& =O\left(\frac{1}{\pi P_{r}} \int_{1 / r}^{\delta} \frac{\phi(t)}{t^{2}} p_{\tau} d t\right) . \\
& \left(\sum_{m=\tau+1}^{\infty} p_{m} \sin (m+1 / 2) t=O\left\{p_{\tau} / t\right\}, \text { for } p_{m} \geq p_{m+1}\right) .
\end{aligned}
$$

Now using (3.2) and (4.1) and using integration by parts, we have 


$$
\begin{aligned}
I_{2,2} & =O\left[\frac{1}{\pi P_{r}}\left(\left[\frac{\Phi(t) p(1 / t)}{t^{2}}\right]_{1 / r}^{\delta}-\int_{1 / r}^{\delta} \Phi(t)\left(\frac{d}{d t} \frac{1}{t \prod_{m=1}^{k} l_{m}(1 / t)}\right) d t\right)\right] \\
& =O\left[\frac{1}{\pi P_{r}}\left(\left\{o\left(\frac{p(1 / t)}{t}\right)\right\}_{1 / r}^{\delta}+\int_{1 / r}^{\delta} \frac{\Phi(t)}{t^{2} \prod_{m=1}^{k} l_{m}(1 / t)}\right)\right] \\
& -O\left[\frac{1}{\pi P_{r}}\left(\int_{1 / r}^{\delta} \frac{\Phi(t)}{t} \frac{d}{d t}\left(\frac{1}{\prod_{m=1}^{k} l_{m}(1 / t)}\right) d t\right)\right] .
\end{aligned}
$$

Furthermore, since, $k$ is fixed, so we can write $P_{r}=l_{k+1}(r)=l(r)$.

Clearly, we have

$$
\begin{aligned}
I_{2,2}= & o\left(v_{r}\right)+o(1)+o\left(\frac{1}{\pi l(r)}\right) \\
& \times\left(\int_{1 / r}^{\delta} \frac{1}{t \prod_{m=1}^{k} l_{m}(1 / t)} d t+\int_{1 / r}^{\delta} \frac{d}{d t}\left\{\frac{1}{\prod_{m=1}^{k} l_{m}(1 / t)}\right\} d t\right) \\
= & o(1)+o\left(\frac{1}{\sqrt{l(r)}}\right) \int_{1 / r}^{\delta}\left(\frac{1}{\left\{t \prod_{m=1}^{k} l_{m}(1 / t)\right\} \sqrt{l(1 / t)}}\right) d t \\
& +o\left\{\frac{1}{l(r)}\left[\frac{1}{\prod_{m=1}^{k} l_{m}(1 / t)}\right]_{1 / r}^{\delta}\right\} \\
= & o(1)+o(\sqrt{l(r)} / \sqrt{l(r)})+o(1 / l(r)) \\
= & o(1) \quad\left(\operatorname{using}(4.1) \text { and } p_{n} \geq p_{n+1}\right)
\end{aligned}
$$

Which completes the proof of Lemma 2.

\section{Proof of Main Theorem}

Following Zygmund [[21], p. 50], we have

$$
\begin{aligned}
T_{r}(x)-s & =\sum_{m=0}^{r} \frac{p_{m}}{P_{r}}\left\{s_{m}(x)-s\right\} \\
& =\sum_{m=0}^{r} \frac{p_{m}}{P_{r}}\left(\frac{1}{\pi} \int_{0}^{\pi} \phi(t) \frac{\sin (m+1 / 2) t}{\sin t / 2}\right) d t \\
& =\sum_{m=0}^{r} \frac{p_{m}}{P_{r}}\left\{\int_{0}^{1 / r}+\int_{1 / r}^{\delta}+\int_{\delta}^{\pi}\right\} \phi(t) \frac{\sin (m+1 / 2) t}{\sin t / 2} d t \\
& =I_{1}+I_{2}+I_{3} \text { (say). }
\end{aligned}
$$

In order to establish the theorem, it is enough to prove,

$$
\sum_{r=0}^{n}\left|T_{r}(x)-s\right|^{2}=o(n) \quad(n \rightarrow \infty) .
$$

By Lemma 1 and Lemma 2, we have 


$$
\begin{aligned}
\left|T_{r}(x)-s\right|= & \frac{1}{\pi P_{r}} \int_{1 / r}^{\delta} \phi(t) \alpha(t) d t+\frac{1}{\pi P_{r}} \int_{1 / r}^{\delta} \phi(t) \beta(t) \cot (t / 2) d t+o(1) \\
\leq & \frac{1}{\pi P_{r}} \int_{1 / n}^{\delta} \phi(t) \alpha(t) d t \\
& +\frac{1}{\pi P_{r}} \int_{1 / n}^{\delta} \phi(t) \beta(t) \cot (t / 2) d t+o(1)(r \leq n) .
\end{aligned}
$$

Since, $\alpha(t)=O\left(P_{\tau}\right)=\beta(t)$, using monotonicity of $[l(1 / t)]^{-1}$, we have

$$
\begin{aligned}
\left|\frac{1}{\pi P_{r}} \int_{1 / n}^{1 / r} \phi(t) \alpha(t) d t\right|= & O\left(\frac{1}{\pi P_{r}} \int_{1 / n}^{1 / r}|\phi(t)| P(1 / t)\right) d t \\
= & O\left(\frac{1}{\pi l(r)}\right) \int_{1 / n}^{1 / r}|\phi(t)| l(1 / t) d t \\
= & O\left(\frac{1}{\pi l(r)}\right)\left[(\Phi(t) l(1 / t))_{1 / n}^{1 / r}-\int_{1 / n}^{1 / r} \frac{\Phi(t) d t}{t \prod_{m=1}^{k} l_{m}(1 / t)}\right] \\
= & O\left(\frac{1}{l(r)}\right)\left[o\left(\frac{t l(1 / t)}{\sqrt{\prod_{m=1}^{k+1} l_{m}(1 / t)}}\right)^{1 / r}\right] \\
& +O\left(\frac{1}{l(r)}\right)\left[\int_{1 / n}^{1 / r} o\left(\frac{t}{\sqrt{\prod_{m=1}^{k+1} l_{m}(1 / t)}}\right) \frac{d t}{t \prod_{m=1}^{k} l_{m}(1 / t)}\right] \\
= & o\left(\frac{1}{r}\right)+o\left(\frac{1}{l(r)\left\{\prod_{m=1}^{k} l_{m}(r)\right\}^{3 / 2}}\right) \int_{1 / n}^{1 / r} d t=o(1) .
\end{aligned}
$$

Similarly,

$$
\left|\frac{1}{\pi P_{r}} \int_{1 / r}^{1 / n} \phi(t) \beta(t) \cot (t / 2) d t\right|=o(1) .
$$

Now, using Minkowskis' inequality, we get

$$
\begin{aligned}
& \left\{\sum_{r=0}^{n}\left|T_{r}(x)-s\right|^{2}\right\}^{\frac{1}{2}} \\
& \leq\left[\sum_{r=0}^{n}\left\{\frac{1}{\pi P_{r}}\left(\int_{1 / r}^{\delta} \phi(t) \alpha(t) d t+\frac{1}{\pi P_{r}} \int_{1 / r}^{\delta} \phi(u) \beta(u) \cot (u / 2) d t\right)\right\}\right]^{1 / 2} \\
& \quad \times\left[\sum_{r=0}^{n}\left\{\frac{1}{\pi P_{r}}\left(\int_{1 / r}^{\delta} \phi(u) \alpha(u) d u+\frac{1}{\pi P_{r}} \int_{1 / r}^{\delta} \phi(u) \beta(u) \cot (u / 2) d u\right)\right\}\right]^{1 / 2} \\
& \quad+o(n)^{1 / 2} \\
& =\left[j_{1}+j_{2}+j_{3}+j_{4}\right]^{1 / 2}+o(n)^{1 / 2} .
\end{aligned}
$$


Let

$$
j_{1}=\frac{1}{\pi^{2}} \int_{1 / n}^{\delta} \int_{1 / n}^{\delta} \phi(t) \alpha(t) \phi(u) \alpha(u)\left\{\sum_{r=0}^{n} \frac{1}{P_{r}^{2}}\right\} d t d u .
$$

As we have,

$$
\begin{aligned}
& \int_{1 / n}^{\delta}|\phi(t)| l(1 / t) d t=[\Phi(t) l(1 / t)]_{1 / n}^{\delta}+\int_{1 / n}^{\delta} \Phi(t) \frac{1}{t \prod_{m=1}^{k} l_{m}(1 / t)} d t \\
& =o\left(\frac{t l(1 / t)}{\sqrt{\prod_{m=1}^{k+1} l_{m}(1 / t)}}\right)_{1 / n}^{\delta}+\int_{1 / n}^{\delta}\left(\frac{1}{\sqrt{\prod_{m=1}^{k+1} l_{m}(1 / t)}}\right)\left(\frac{1}{\prod_{m=1}^{k} l_{m}(1 / t)}\right) d t \\
& =o\left(\frac{t l(1 / t)}{\sqrt{\prod_{m=1}^{k+1} l_{m}(1 / t)}}\right)_{1 / n}^{\delta}+\left(\frac{1}{\sqrt{\prod_{m=1}^{k+1} l_{m}(1 / t)} \prod_{m=1}^{k} l_{m}(1 / t)}\right) \int_{1 / n}^{\delta} d t \\
& =o(1) .
\end{aligned}
$$

So, for $\alpha(t)=O\left(P_{\tau}\right)$, and by using (3.1), we get

$$
j_{1} \leq \sum_{r=0}^{n} \frac{1}{P_{r}^{2}} \int_{1 / n}^{\delta}|\phi(t)| l(1 / t) d t \int_{1 / n}^{\delta}|\phi(u)| l(1 / u) d u=o(1) .
$$

Next, as

$$
\begin{aligned}
\int_{1 / n}^{\delta}|\phi(u)| \alpha(u)|\cot (u / 2)| d u= & \int_{1 / n}^{\delta} \frac{|\phi(u)| \alpha(u)}{u} d u \\
= & {\left[\Phi(u) \frac{l(u)}{u}\right]_{1 / n}^{\delta}-\int_{1 / n}^{\delta}\left[\Phi(u) \frac{d}{d u}\left(\frac{l(1 / u)}{u}\right)\right] d u } \\
= & {\left[\Phi(u) \frac{l(u)}{u}\right]_{1 / n}^{\delta}+\int_{1 / n}^{\delta} \Phi(u) \frac{1}{u}\left(\frac{1}{u \prod_{m=1}^{k} l_{m}(1 / u)}\right) d u } \\
= & \left.o\left(\frac{l(1 / u)}{\sqrt{\prod_{m=1}^{k+1} l_{m}(1 / u)}}\right)_{1 / n}^{\delta}\right) \\
& +o\left(\frac{1}{\left(\sqrt{\prod_{m=1}^{k+1} l_{m}(1 / u)}\right)\left(u \prod_{m=1}^{k} l_{m}(1 / u)\right)}\right) \int_{1 / n}^{\delta} d u \\
= & o(1) ;
\end{aligned}
$$

so it implies 


$$
\begin{aligned}
j_{2} & =\frac{1}{\pi^{2}} \int_{1 / n}^{\delta} \int_{1 / n}^{\delta} \phi(t) \alpha(t) \phi(u) \beta(u) \cot (u / 2)\left\{\sum_{r=0}^{n} \frac{1}{P_{r}^{2}}\right\} d t d u \\
& \leq \frac{1}{\pi^{2}} \sum_{r=0}^{n} \frac{1}{P_{r}^{2}}\left(\int_{1 / n}^{\delta}|\phi(t)| \alpha(t) d t \int_{1 / n}^{\delta}|\phi(u)| \alpha(u)|\cot (t / 2)| d u\right) \\
& =o(1) \int_{1 / n}^{\delta}|\phi(t)| \alpha(t) d t \int_{1 / n}^{\delta}|\phi(u)| \alpha(u)|\cot (t / 2)| d u \\
& =o(1) .
\end{aligned}
$$

Similarly, we can obtain

$$
j_{3}=o(1) \quad \text { and } \quad j_{4}=o(1) .
$$

Hence, the proof of the theorem is completed.

Corollary 1. If the condition (3.1) of main theorem and the condition (1.8) of [12] are satisfied, then the series $\sum_{n=0}^{\infty} u_{n}(t)$ is $[C, 1,2]$ - summable to the sum $s$ for $t=x$.

\section{References}

[1] A. A. Das, B. B. Jena, S.K. Paikray and R. K. Jati, "Statistical deferred weighted summability and associated Korovkin-type approximation theorem", Nonlinear sciences letters A, vol. 9, no. 3, pp. 238-245, 2018.

[2] M. Fekete, "Vizsagàlatok a Fourier-sorokol", Mathematikai és Természettudományi Értesítő, vol. 34, pp. 759-786, 1916.

[3] G. H. Hardy and J. E. Littlewood, "Sur la série de Fourier d'une fonction a carré sommable", Comptes Rendus de l'Académie des Sciences - Series I - Mathematics, vol. 156, pp. 1307-1309, 1913.

[4] J. M. Hyslop, "Note on the strong summability of series", Glasgow mathematical journal, vol. 1, no. 1, pp. 16-20, Jan. 1951, doi: $10.1017 /$ S2040618500032883

[5] B. B. Jena and S. K. Paikray, "Product of statistical probability convergence and its applications to Korovkin-type theorem", Miskolc mathematical notes, vol. 20, no. 2, pp. 969-984, 2019, doi: 10.18514/ MMN.2019.3014 
[6] B. B. Jena, S. K. Paikray, and H. Dutta, "On various new concepts of statistical convergence for sequences of random variables via deferred Cesàro mean", Journal mathematical analysis and applications, vol. 487, no. 1, Art. ID 123950, Jul. 2020, doi: 10.1016/j.jmaa.2020.123950

[7] B. B. Jena, Vandana, S. K. Paikray, and U. K. Misra, "On generalized local property of $|\mathrm{A} ; \delta| \mathrm{k}$-summability of factored Fourier series", International journal of analysis and applications, vol. 16, no. 2, pp. 209-221, 2018. [On line]. Available: https:/ / bit.ly/ 359BDsx

[8] B. B. Jena, S. K. Paikray, and U. K. Misra, "A Tauberian theorem for double Cesàro summability method", International journal of mathematics and sciences, Art. ID. 2431010, 2016, doi: $10.1155 / 2016 / 2431010$

[9] B. B. Jena, S. K. Paikray, and U. K. Misra, "Inclusion theorems on general convergence and statistical convergence of $(L, 1,1)$-summability using generalized Tauberian conditions", Tamsui Oxford journal of infinity mathematical sciences, vol. 31, no. 1, pp. 101-115, 2017. [On line]. Available: https:/ / bit.ly/ 38nxTFQ

[10] B. B. Jena, S. K. Paikray, and U. K. Misra, "Statistical deferred Cesàro summability and its applications to approximation theorems", Filomat, vol. 32, no. 6, pp. 2307-2319, 2018, doi: 10.2298/ FIL1806307J

[11] B. B. Jena, L. N. Mishra, S. K. Paikray, and U. K. Misra, "Approximation of signals by general matrix summability with effects of Gibbs phenomenon", Boletim Sociedade Paranaense de Matemática, vol. 38, no. 6, pp. 141-158, 2020, doi: 10.5269/ bspm.v38i6.39280

[12] M. L. Mittal, "A Tauberian theorem on strong Nörlund summability", Journal of Indian Mathematical Society, vol. 44, pp. 369-377, 1980.

[13] M. L. Mittal, "On strong Nörlund summability of Fourier series", Journal of mathematical analysis applications, vol. 314, no. 1, pp. 75-84, Feb. 2006, doi: 10.1016/j.jmaa.2005.01.072

[14] M. L. Mittal and R. Kumar, "A note on strong Nörlund summability", Journal of mathematical analysis applications, vol. 199, no. 1, pp. 312-322, Apr. 1996, doi: 10.1006/jmaa.1996.0143

[15] P. Parida, S. K. Paikray, H. Dutta, B. B. Jena, and M. Dash, "Tauberian theorems for Cesàro summability of nth sequences", Filomat, vol. 32, no. 11, 2018, doi: 10.2298/ FIL1811993P 
[16] T. Pradhan, S. K. Paikray, B. B. Jena, and H. Dutta, "Statistical deferred weighted B-summability and its applications to associated approximation theorems", Journal of inequality and applied, vol. 2018, Art. ID: 65, Mar. 2018, doi: 10.1186/ s13660-018-1650-x

[17] H. M. Srivastava, B. B. Jena, S. K. Paikray, and U. K. Misra, "A certain class of weighted statistical convergence and associated Korovkin type approximation theorems for trigonometric functions", Mathematical methods in the applied sciences, vol. 41, no. 2, pp. 671-683, Jan. 2018, doi: 10.1002/ mma.4636

[18] H. M. Srivastava, B. B. Jena, S. K. Paikray, and U. K. Misra, "Generalized equi-statistical convergence of the deferred Nörlund summability and its applications to associated approximation theorems", Revista de la Real Academia de Ciencias Exactas, Físicas y Naturales. Serie A. Matemáticas, vol. 112, pp. 1487-1501, Oct. 2018, doi: 10.1007/ s13398-017-0442-3

[19] H. M. Srivastava, B. B. Jena, S. K. Paikray, and U. K. Misra, "Deferred weighted A-statistical convergence based upon the ( $p, q)$-Lagrange polynomials and its applications to approximation theorems", Journal of applied analysis, vol. 24, no. 1, pp. 1-16, May 2018, doi: 10.1515/ jaa-2018-0001

[20] S. K. Paikray, B. B. Jena, and U. K. Misra, "Statistical deferred Cesàro summability mean based on ( $p, q)$-integers with application to approximation theorems," in Advances in summability and approximation theory, S. A. Mohiuddine and T. Acar, Eds. Singapore: Springer, 2018, pp. 203-222, doi: 10.1007/ 978-981-13-3077-3_13

[21] A. Zygmund, Trigonometric series, 2nd ed., vol. 1. Cambridge: Cambridge University Press, 1959. 